\title{
What if You Cannot See the Target? Two Case Studies of Atlas-to-Patient Registration in Trigeminal Neuralgia Radiosurgery.
}

\author{
Joseph Stancanello $^{1}$, Alexander Muacevic ${ }^{2}$ \\ 1. GE Healthcare 2. European CyberKnife Center Munich
}

$\square$ Corresponding author: Joseph Stancanello, joseph.stancanello@polimi.it

Disclosures can be found in Additional Information at the end of the article

\section{Abstract}

Introduction: Medically resistant trigeminal neuralgia (TN) is a well-suited pathology to be treated by radiosurgery (RS). A significant percentage (60\%-70\%) of patients is expected to benefit from the RS treatment. Imaging is crucial to identify the target, though unfortunately in some cases the trigeminal nerve could not be visible even in high field magnetic resonance images (MRI). The aim of this work is to develop and relatively validate a method for the atlasbased identification of the target in those cases where the trigeminal nerve is not visible.

Material Methods: The proposed method is based on the modification of a previously published approach for functional RS, adapted to the TN. A high resolution, high contrast volumetric T1MRI atlas was non-rigidly registered to patient T1-MRI. Two patients scanned with a 3T T1weighted-MRI and trigeminal nerve specific MR (Constructive Interference in Steady State CISS) sequences were considered in this study. In both cases, the right-sided trigeminal nerve was not visible, while it was the contralateral nerve. For one patient, we simulated a potential radiosurgical treatment plan. The atlas-based localization accuracy of the trigeminal nerve was validated in a relative way evaluating the nearby structure continuity and the contralateral nerve localization.

Results: The atlas-based fusion showed a high degree of relative accuracy in both cases, suggesting a not ideal coverage of the trigeminal nerve in the simulated treatment plan while opening the way to a possible treatment for both patients.

Conclusions: The proposed method holds potential to support the physician to correctly locate the target in those cases where trigeminal nerve is not visible for RS, as well as other therapeutic image-guided applications. Additional cases and further validation in absolute way are needed to definitively assess the accuracy of the method.

Published 11/30/2010

C) Copyright 2010

Stancanello et al. This is an open access article distributed under the terms of the Creative Commons Attribution License CC-BY 3.0., which permits unrestricted use, distribution, and reproduction in any medium, provided the original author and source are credited.
Categories: Neurosurgery

Keywords: contralateral nerve, ciss-mri, functional radiosurgery, image-guided therapy, trigeminal neuralgia

\section{Introduction}

Trigeminal neuralgia (TN) is a neuropathic disorder of the fifth cranial nerve. It can be idiopathic but also related to other pathologies (e.g., multiple sclerosis) or injuries. No medical 
test exists that clearly diagnoses all cases of TN: however, the relief of pain by carbamazepine gives more credit to the diagnosis of TN. This pathology is a well-suited treatment indication for frame-based or frameless radiosurgery in selected patients suffering from the typical facial pain syndrome. This is described as attacks with abrupt in onset with lancinating characteristics. Radiosurgery may be used as an alternative to microvascular decompression or thermo-coagulation and as a coadjuvant to anticonvulsants in order to reduce the amount of required medication [1-4]. The exact mechanism of pain relief through radiosurgery is currently unknown. It relies on two steps: 1) immediate decrease in the intensity of the pain even if the attacks still occur (immediate interruption of ephaptic transmission) and 2) complete cessation of the attacks several weeks later (delayed demyelination injury to the nerve). The actual maximum dose ranges from 80 to $100 \mathrm{~Gy}$, where nerve necrosis has been identified when delivering 100 Gy. Dedicated radiosurgical devices, such as the Gamma Knife (Elekta AB, Stockolm, Sweden) or the CyberKnife (Accuray Inc., Sunnyvale, USA) as well as conventional linear accelerators (LINACs) equipped with radiosurgical accessories [5], have been successfully utilized to treat trigeminal neuralgia. Several reports document long-term pain relief after focal irradiation in $70-80 \%$ of patients through frame-based or frameless radiosurgery [6-8].

Enhanced imaging techniques play a crucial role and may facilitate target delineation and result in wider clinical applicability. Exact target definition is of utmost importance, as not only the applied dose to the nerve, but also the volume of the irradiated nerve, directly affect pain relief and potential adverse reactions, such as facial dysesthesias or numbness [9]. The high prescription dose to manage trigeminal neuralgia calls for high accuracy in target delineation in order to minimize the possible side-effects without invalidating the success rate of the treatment. In order to meet this clinical need, several approaches have been applied to the accurate visualization of the nerve in all its segments. Computed tomography (CT) is not able to offer the soft tissue contrast suitable for a clear identification of the trigeminal nerve; while sporadically the nerve may be partially visualized, in the majority of the cases it is often invisible. CT cisternography has been utilized to visualize the segment of the nerve to be treated [9]. Due to the need for the injection of contrast agent to highlight the cistern, this approach has not been widely applied in the clinical centers. Magnetic resonance imaging (MRI) may be considered the best imaging modality to clearly distinguish the trigeminal nerve due to its high soft tissue contrast. The wide adoption of 1.5T MRI scanners has facilitated the possibility of using this modality also for trigeminal neuralgia purposes. Also, the recent introduction of 3T MRI scanners in combination with specialized sequences, such as fast imaging employing steady-state acquisition (FIESTA) [10] and three-dimensional constructive interference in steady state (CISS) [11], promised to pave the road to an easy, clear identification of the trigeminal nerve in all its segments. Unfortunately, even using thin cut (0.7 - $1.0 \mathrm{~mm}) \mathrm{T} 1$ and T2 MRI studies nowadays widely applied, the definition of the full outline of the nerve in its cisternal compartment along the retrogasserian trigeminal root is a complex task. This applies specifically to patients with recurrent pain syndromes with documented surgical procedures, as the nerve might be atrophic and therefore difficult to identify. Moreover, there might be significant variations in patient's local anatomy and morphology, which should be accounted for in order to optimize outcome and reduce the complication rate [12]. Additionally, only a few centers are currently equipped with 3T MRI scanners and a small fraction of patients affected by TN may not be suitable for MRI due to the presence of prostheses or pacemakers. In such cases, CT cisternography has been reported to provide good results [13].

Our group has recently paid particular attention to the development of a method for applications in functional radiosurgery [14]: the approach was focused on adding atlas information to the patient MRI, with regards to several brain nuclei. In an attempt to better elucidate also the anatomical localization of the trigeminal nerve in this work, we develop a frame to enhance the delineation accuracy through atlas-based information in several conditions calling for additional information. The following cases are examples of possible 
applications of the proposed method:

A. atlas-based information addition to patient 1.5T MRI, in those cases where the trigeminal nerve is not clearly identifiable at all or in all its segments and no 3T scanner is available;

B. atlas-based information addition to patient 3T MRI, in those cases where the trigeminal nerve is not clearly identifiable at all or in all its segments.

Translating these tasks from clinical perspective into technical specifications implies the need for mono-modality deformable registration of atlas to patient MRI. So far, the deformable registration process has been considered time-consuming and therefore adopted only in a few research centers, while wide clinical applications are still missing. On the basis of the current trend to shorten the registration time $[15,16]$, we focus on developing a solution fast enough to be widely adoptable not only in research but also in clinical centers.

The aim of this work is to tailor the method already proposed for atlas-based functional radiosurgery to the special case of TN treatment, to be applied to those cases where target is not directly visible, even in high field scans acquired with trigeminal nerve identification specific sequences. Treatment planning for standard image-guided treatments like microvascular decompression or thermocoagulation are expected to benefit as well from the application of the proposed method.

\section{Case Presentation}

\section{Patient and imaging data}

MRIs have been extensively used for the localization of trigeminal nerve $[17,18]$ due to the possibility of directly visualizing the nerve as well as the possible vascular compression. Both morphological MRI and magnetic resonance angiography (MRA) [19] have been widely proposed to understand the aetiology of the trigeminal neuralgia and plan the appropriate therapeutic strategy. Additionally, CT scans have been suggested to offer complementary information, especially for the bony structures [20] and provide information able to reduce the uncertainty present in the localization of the nerve when using MRI only [21]. Therefore, to localize the trigeminal nerve in functional radiosurgery, two patients affected by trigeminal neuralgia and candidates scheduled to undergo trigeminal radiosurgery by the CyberKnife were imaged with a 3T scanner (TrioTim, Siemens Healthcare, Erlangen, Germany) using several T1weighted (T1w) and T2-weighted (T2w) pulse sequences with different parameters. The trigeminal nerve specific sequence CISS - producing T2w-MRI - was also used in this study. Acquisition and reconstruction parameter set-up used in this study is reported in Table 1. Number of excitations (NEX) was equal to one for both studies. In both cases, the direct visualization of the trigeminal nerve was not possible. T1w-MR images were used for the automatic local non-rigid (LNR) registrations of the Montreal Neurological Institute (MM) electronic atlas onto patient volumes, according to the method already described in our previous work [22] and briefly described in the next paragraph. 


\section{Cureus}

\begin{tabular}{|c|c|c|}
\hline Parameter & Value & \\
\hline Scan sequence & T1w & CISS \\
\hline Slice orientation & Coronal & Axial \\
\hline TR & $9.3 \mathrm{~ms}$ & $5.89 \mathrm{~ms}$ \\
\hline TE & $4.92 \mathrm{~ms}$ & $2.47 \mathrm{~ms}$ \\
\hline Echo train & 1 & 1 \\
\hline Flip angle & 20 & 43 \\
\hline Slice thickness & $1 \mathrm{~mm}$ & $0.8 \mathrm{~mm}$ \\
\hline In-plane resolution & $0.6^{\star} 0.6 \mathrm{~mm} 2$ & $0.5^{\star} 0.5 \mathrm{~mm} 2$ \\
\hline Matrix & $220 * 320$ & $384^{\star} 348$ \\
\hline
\end{tabular}

TABLE 1: Acquisition parameters and spatial resolution of the acquired Tlw- and CISS-MRIs, second and third columns, respectively.

\section{Atlas-to-patient registration technique}

All the registrations were performed in MATLAB (MATLAB, The Mathworks, Inc., Natick, MA). CISS-MRI volume was rigidly registered to the corresponding T1w-MRI for both patient by means of a mutual information based algorithm [23], whose objective function consisting of mutual information; the latter was optimized using a multiresolution strategy in which the first optimization employed $4 \mathrm{~mm}$ sampling, followed by a second one with $2 \mathrm{~mm}$ sampling which used the result of the first step as the starting point. Optimization was based on the BrentPowell method [24]. The MM standard brain volume (or atlas) was then registered onto each T1w-MR patient image, following a method and parameter setup already described in our previous work [22]. This consists of two affine global transformations (the whole volume and the region-of-interest - ROI - registrations) followed by local non-rigid (LNR) registration: while affine registrations are based on a normalized mutual information algorithm, the LNR registrations consist of the optimization of a two-part objective function made of the weighted sum of a similarity criterion and a regularization term [25] in order to match the floating MM electronic standard brain onto the reference patient T1w-MRI volume. Voxels were grouped by eight, forming $2 * 2 * 2$ cubes. The volume contained in these cubes was divided into five irregular tetrahedra, whose vertices are placed in the center of each voxel. The mesh created by the tetrahedra covered the volume of the image. The vertices were taken as control points which were moved in order to minimize the squared differences of the intensities between corresponding voxels. This represented the similarity criterion between the datasets. Within each tetrahedron, the deformation was modeled by an affine transformation resulting in displacement of the vertices. Such minimization of differences leads to a non-smooth deformation field, which does not necessarily preserve continuity. Therefore, another term was added to the similarity criterion intended to create a smooth deformation field. This regularization term is based on the change of volume of each tetrahedron, calculated by means of the Jacobian of the transformation. The effect of the regularization term on the objective function was modulated using a regularization factor which sets the trade-off between the deformation of the image and the smoothness of the deformation field. The objective function 
was optimized by applying a steepest descent algorithm, which searches for the local optimum solution in the neighbourhood of the initial conditions, which are supposed to be favourable because of the previously applied global and local affine transformations. The regularization factor, as in our previous works [14-22], was initially set equal to 0.4 , which provided the most accurate matching of the brain structures with a LNR registration error smaller than or equal to the maximum voxel size (mvs) of the datasets to be registered. Different regularization factors were investigated for special cases of dramatic changes in the brain geometry, but the initial value proved to offer the most accurate match

After the global affine registration, all images (T1w-MRI, CISS-MRI and MNI) were cropped to obtain ROIs with size equal to $40 * 40 * 40 \mathrm{~mm} 3$ around the trigeminal nerve region; hence, a second affine registration was performed, taking into account only the MM and patient T1wMRI ROIs. The combination of cropping images and reregistering based on just the ROIs had the effect of speeding up the time-consuming LNR registration, allowing the overall procedure to be performed in less than $10 \mathrm{~min}$ (LNR less than $5 \mathrm{~min}$ ) on a personal computer (PC) equipped with a $3 \mathrm{GHz}$ central process unit (CPU) and $1.5 \mathrm{MB}$ random access memory (RAM). Moreover, this approach allowed the maximum number of iterations in LNR registration to be reduced to 32, due to the high similarity of the volumes at the end of the second affine registration. All registration results were visually inspected in order to evaluate the continuity of anatomical structures, by means of cross hairs. In particular, the maximum allowable error was chosen equal to $1 \mathrm{~m}$, i.e. the slice thickness of T1w-MRIs. This margin of error is within the limits of localization error for framabased and frameless systems for stereotactic surgical or radiosurgical targeting. Once the deformation field was calculated and evaluated by visual inspection, it was possible to identify the trigeminal nerve on the atlas and superimpose it on the T1w- and CISS-MRIs.

\section{Validation}

Unfortunately, no ground truth was available in order to validate the atlas-based identification of the trigeminal nerve in absolute way. For such a reason we validated the results in a relative way, i.e., evaluating the accuracy of the structures visible in the CISS-MRI and in the atlas. From that we inferred, the accuracy of the trigeminal nerve not directly visible. Additionally, the accuracy of the atlas-based identification was also tested by considering the contralateral trigeminal nerve, which was well identifiable in both cases. It must be noticed that all these validation criteria are not per se representative of the accuracy of the method, because some local abnormalities of the brain may occur and the outcome of the treatment could be poor, even if correctly targeting the nerve due to the physiological response to the treatment varying case by case (see also introduction). Nevertheless, due to the absence of any absolute validation criteria, we have adopted this relative evaluation as a preliminary methodology to assess the quality of our results.

\section{Discussion}

Both described cases are special. It was our aim to show the principal applicability of the atlasbased information to TN stereotactic radiosurgery (SRS) planning in challenging cases. Both patients were suffering from severe TN symptoms, even though they were on high medical treatment regimes.

Patient 1 was treated two times by microvascular decompression in 2003 and by additional thermocoagulation the same year. Additionally, a Gamma Knife procedure was performed in 2004. All applied treatments could not achieve a prolonged and satisfactory pain control; therefore, we analyzed the potential applicability of an additional radiosurgical treatment using CyberKnife SRS. We simulated a treatment plan on standard imaging and then retrospectively matched the atlas-based information to the patient MRIs. Figure 1 shows the correspondence 


\section{Cureus}

between the automatically identified atlas-based trigeminal nerve and the CISS-MRI, for the invisible (right side) as well as visible (left side) nerves. The crosshair indicates an accurate correspondence in the contralateral nerve, which could suggest also high accuracy in the localization of the right, invisible trigeminal nerve. The anatomy of the cisternal nerve compartment was completely distorted due to the already performed treatment approaches.
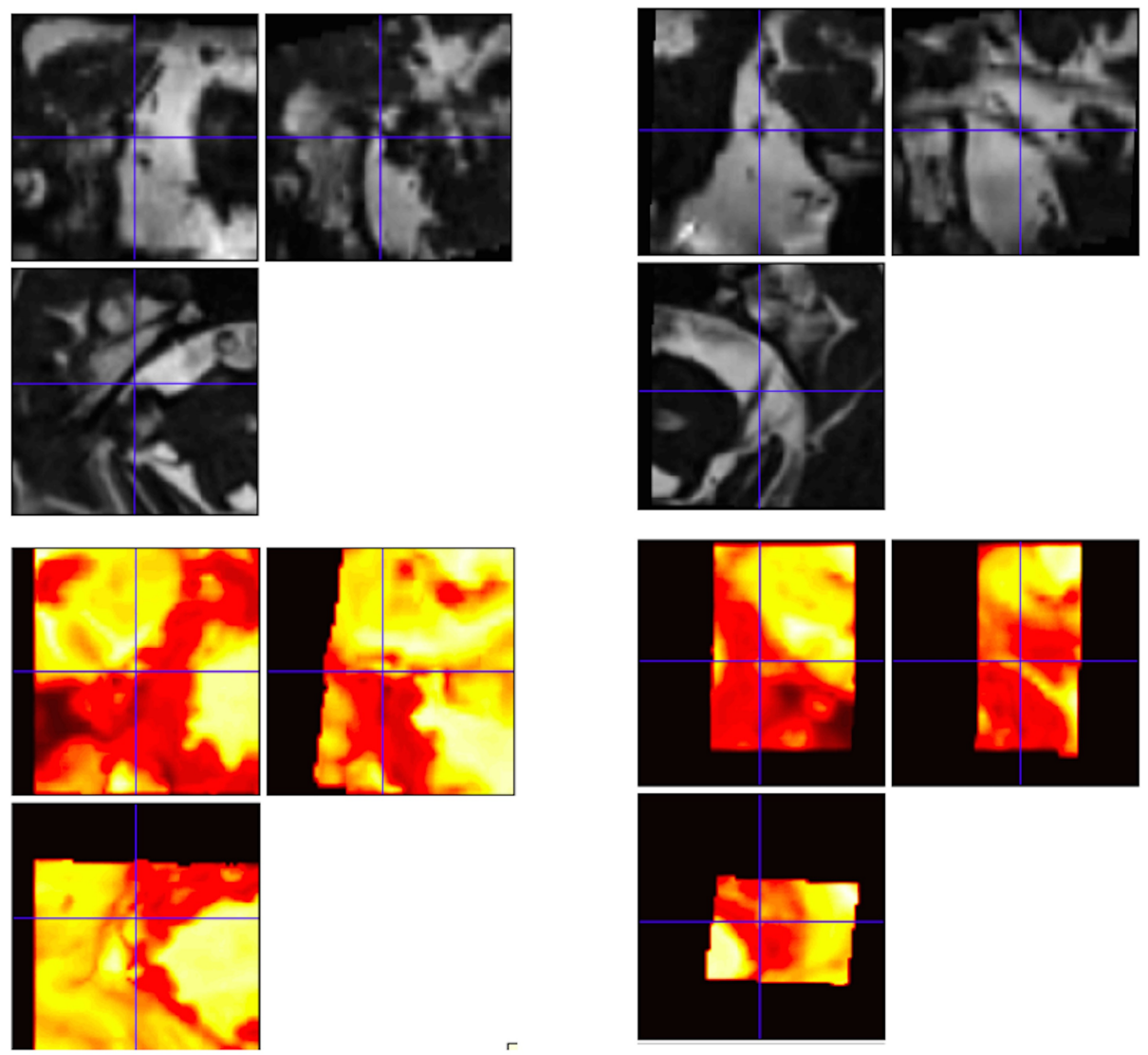

\section{FIGURE 1: Patient 1. Left-column: CISS-MRI (upper-left) geometrical coherent to the MNI atlas (lower-left) to identify the right trigeminal nerve invisible on CISS-MRI.}

Right column: CISS-MRI (upper-right) geometrical coherent to the MNI atlas (lower-right) to identify the contralateral trigeminal nerve visible on CISS-MRI. Crosshair indicates in both cases the relationship between the automatically identified trigeminal nerve and the correspondent location onto the CISS MRI.

We retrospectively have matched the atlas-based information to our planning target volume and could detect overlap but not ideal coverage of atlas and planning MRI information (Figure 2). Patient 2 was also treated by thermocoagulation in 2003 but was still on high Lyrica and carbamazepin medication because of no symptom improvement. We were investigating to plan SRS on the right-sided nerve but could not detect the nerve in its cisternal aspect. Therefore, treatment was not performed so far, but as the here-described planning method evolves, we might see a chance for a second SRS approach in the future. In fact, as shown in Figure 3, accurate correspondence was found in the controlateral trigeminal nerve (left side) suggesting an accurate localization also of the right trigeminal nerve. The right-sided nerve seems to be far lateral with a very unusual course through the cisternal compartment. 


\section{Cureus}
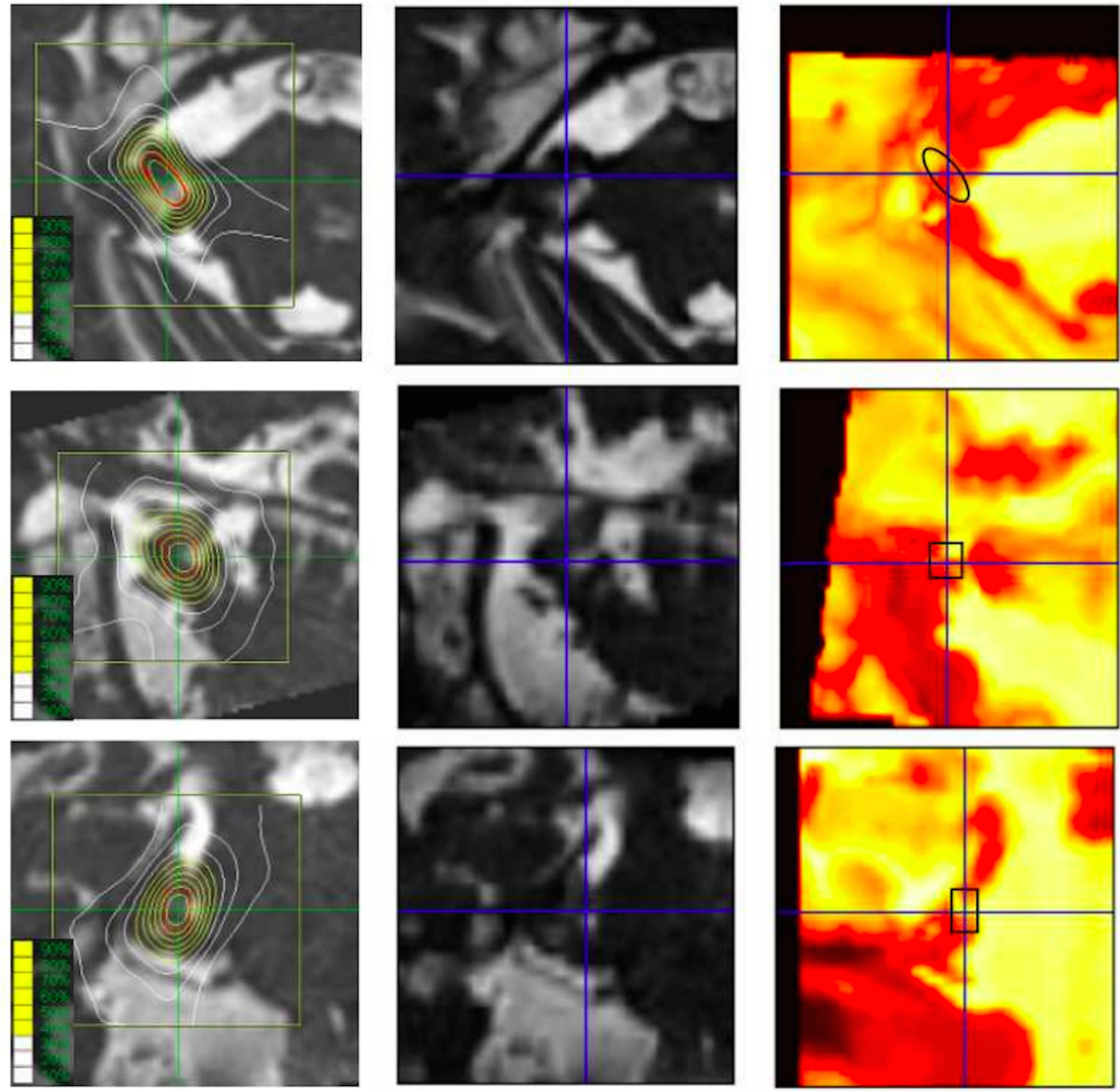

\section{FIGURE 2: Patient 1. Left-column: Target (red line) and isodose}

\section{lines of the radiosurgical treatment simulated by the}

\section{CyberKnife.}

Patient 1. Left-column: Target (red line) and isodose lines of the radiosurgical treatment simulated by the CyberKnife, superimposed on axial (top), sagittal (middle) and coronal (bottom) views of CISS-MRI. Central-column: CISS-MRI used to localize the correspondence between the atlas-based and the manually identified targets. Right-column: atlas-based identification of the right trigeminal nerve. Black lines show the manually delineated target from left column superimposed on the atlas registered to the patient T1w-MRI. Notice the suboptimal manual identification of the target. 


\section{Cureus}
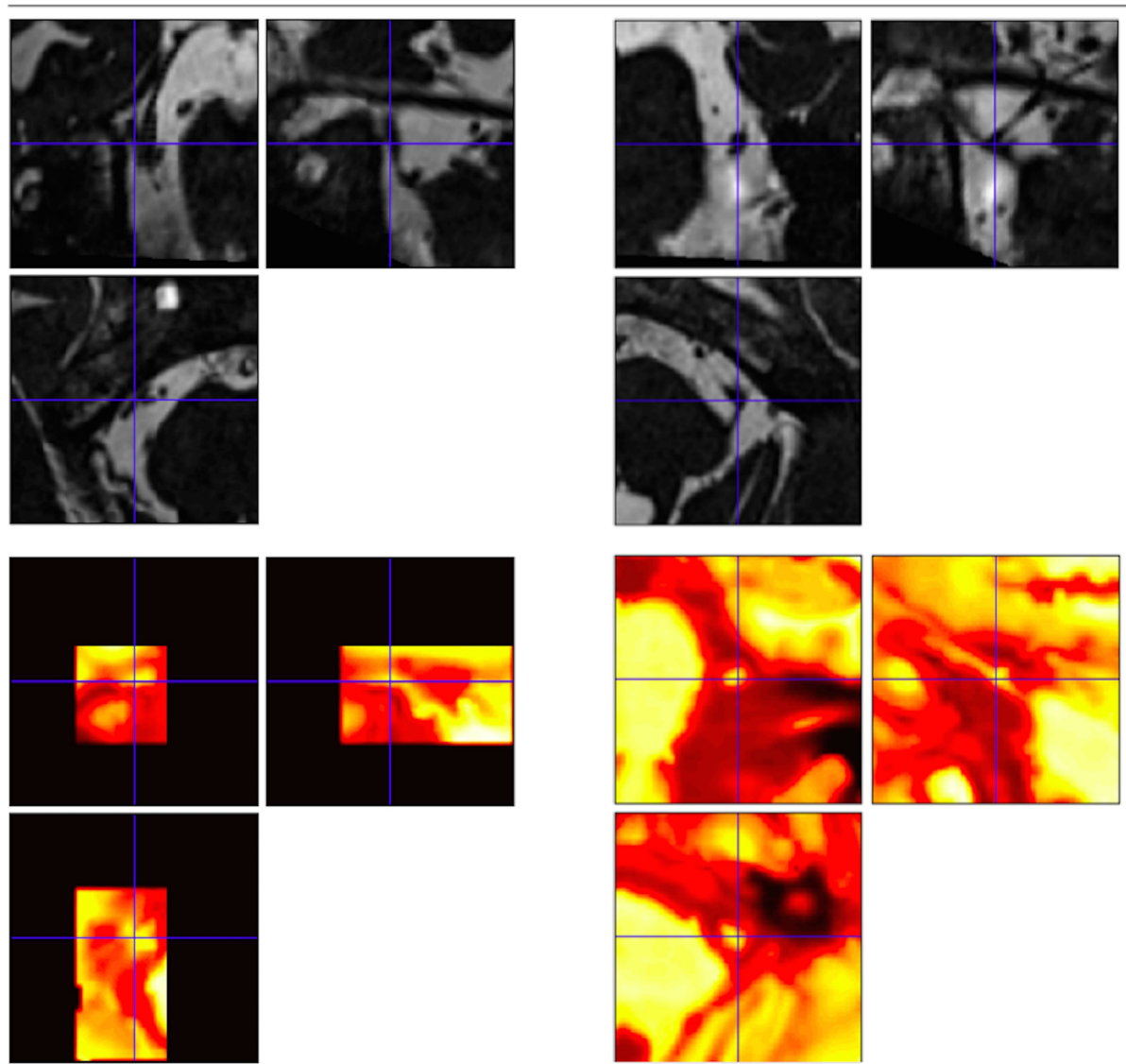

FIGURE 3: Patient 2. Left-column: CISS-MRI (upper-left) geometrical coherent to the MNI atlas (lower-left) to identify the right trigeminal nerve.

Patient 2. Left-column: CISS-MRI (upper-left) geometrical coherent to the MNI atlas (lower-left) to identify the right trigeminal nerve invisible on CISS-MRI. Right column: CISS-MRI (upperright) geometrical coherent to the MNI atlas (lower-right) to identify the contralateral trigeminal nerve visible on CISS-MRI. Crosshair indicates in both cases the relationship between the automatically identified trigeminal nerve and the correspondent location onto the CISS MRI.

A possible absolute validation of the method could consist of registering 1.5T MRIs where trigeminal nerve is not visible and 3T MRIs of the same patients, where hopefully the trigeminal nerve is visible. The here proposed method would be applied to the former dataset, while the latter would be used to validate the atlas-based identification, once the geometrical coherence is established over all the datasets.

We want to clearly point out that it is absolutely necessary to be sure about the location of the $\mathrm{TN}$ nerve on the underlying imaging studies for TN radiosurgery. However, there are very selected cases where no standard treatment application is of help and the clinical symptoms are so disturbing, but the nerve can hardly be detected with conventional imaging techniques. In these cases with inferior identification of the TN, the here described method might offer help to elucidate the nerve better and to gain confidence for a potential $\mathrm{TN}$ treatment options.

\section{Conclusions}


In conclusion, while it is not possible to definitively assess the absolute accuracy of the proposed method, we think it holds a useful potential to help delineate targets in trigeminal neuralgia, especially in those cases where the direct identification is not possible. The method could provide physicians with a fast and reliable tool for robust identification in total absence or poor quality of nerve visualization. A larger number of cases and additional validations are needed to test the robustness and the absolute accuracy of the method.

\section{Additional Information}

\section{Disclosures}

Human subjects: Consent was obtained by all participants in this study. Patients were informed of, and consented to, possible use of their data for research purposes. Conflicts of interest: In compliance with the ICMJE uniform disclosure form, all authors declare the following: Payment/services info: All authors have declared that no financial support was received from any organization for the submitted work. Financial relationships: Joseph Stancanello declare(s) an alternate financial activity from Seimens Healthcare. At that time I was an employee of Seimens Healthcare. Other relationships: All authors have declared that there are no other relationships or activities that could appear to have influenced the submitted work.

\section{References}

1. Flickinger JC, Pollock BE, Kondziolka D, Phuong LK, Foote RL, Stafford SL, Lunsford LD: Does increased nerve length within the treatment volume improve trigeminal neuralgia radiosurgery? A prospective double-blind, randomized study". Int J Radiat Oncol Biol Phys. 2001, 51:449-54.

2. Kondziolka D, Lunsford LD, Flickinger JC, Young RF, Vermeulen S, Duma CM, Jacques DB, Rand RW, Regis J, Peragut JC, Manera L, Epstein MH, Lindquist CJ: "Stereotactic radiosurgery for trigeminal neuralgia: a multiinstitutional study using the gamma unit". Neurosurg. 1996, 84:940-45.

3. Leksell L: "The stereotaxic method and radiosurgery of the brain" . Acta Chir Scand. 1951, 102:316-19.

4. Adler JR Jr, Bower R, Gupta G, Lim M, Efi-on A, Gibbs IC, Chang SD, Soltys SG: "Nonisocentric radiosurgical rhizotomy for trigeminal neuralgia". Neurosurgery. 2009, 64:84-90.

5. Chen JC, Girvigian M, Greathouse H, Miller M, Rahimian J: "Treatment of trigeminal neuralgia with linear accelerator radiosurgery: initial results". J Neurosurg. 2004, 101:346-50.

6. Massager N, Abeloos L, Devriendt D, Op de Beeck M, Levivier M: Clinical evaluation of targeting accuracy of gamma knife radiosurgery in trigeminal neuralgia. Int J Radiat Oncol Biol Phys. 2007, 69:1514-20.

7. Regis J, Metellus P, Hayashi M, Roussel P, Donnet A, Bille-Turc F: "Prospective controlled trial of gamma knife surgery for essential trigeminal neuralgia". J Neurosurg. 2006, 104:913-24.

8. Villavicencio AT, Lim M, Burneikiene S, Romanelli P, Adler JR, McNeely L, Chang SD, Fariselli L, McIntyre M, Bower R, Broggi G, Thramann JJ: "CyberKnife radiosurgery for trigeminal neuralgia treatment: a preliminary multicenter experience". Neurosurgery. 2008, 62:647-55.

9. Lim M, Cotrutz C, Romanelli P, Schaal D, Gibbs I, Chang SD, Adler JR: "Stereotactic radiosurgery using CT cisternography and non-isocentric planning for the treatment of trigeminal neuralgia". Comput Aided Surg. 2006, 11:11-20.

10. Chavez GD, De Salles AA, Solberg TD, Pedroso A, Espinoza D, Villablanca P: "Three. dimensional fast imaging employing steady-state acquisition magnetic resonance imaging for stereotactic radiosurgery of trigeminal neuralgia". Neurosurgery. 2005, 56:628.

11. Zerris VA, Noren GC, Shucart WA, Rogg J, Friehs GM: "Targeting the cranial nerve: microradiosurgery for trigeminal neuralgia with CISS and 3D-flash MR imaging sequences". J Neurosurg. 2005, 102:107-10.

12. Borchers JD 3rd, Yang HJ, Sakamoto GT, Howes GA, Gupta G, Chang SD, Adler JR Jr: "CyberKnife stereotactic radiosurgical rhizotomy for trigeminal neuralgia: anatomic and morphological considerations". Neurosurgery. 2009, 64:91-95. 
13. Worthington C, Hutson K, Boulware R, Neglia W, Gibbons JP, Clark R, Rand J: "Computerized tomography cisternography of the trigeminal nerve for stereotactic radiosurgery. Case report". J Neurosurg.. 2000, 93:169-71.

14. Stancanello J, Romanelli P, Modugno N, Cerveri P, Ferrigno G, Uggeri F, Cantore G: "Atlasbased identification of targets for functional radiosurgery". Med Phys. 2006, 33:1603-11.

15. Sohn M, Birkner M, Chi Y, Wang J, Di Y, Berger B, Alber M: "Model-independent, multimodality deformable image registration by local matching of anatomical features and minimization of elastic energy". Med Phys. 2008, 35:866-78.

16. Kaus MR, Brock KK, Pekar V, Dawson LA, Nichol AM, Jaffi-ay DA: "Assessment of a modelbased deformable image registration approach for radiation therapy planning". Int J Radiat Oncol Biol Phys. 2007, 68:572-80.

17. Borges A, Casselman J: "Imaging the trigeminal nerve". Eur J Radiol. 2010, 74:323-40.

18. Tash RR, Sze G, Leslie DR: "Trigeminal neuralgia: MR imaging features" . Radiology. 1989, 172:767-70.

19. Patel NK, Aquilina K, Clarke Y, Renowden SA, Coakham HB: "How accurate is magnetic resonance angiography in predicting neurovascular compression in patients with trigeminal neuralgia? A prospective, single-blinded comparative study". Br J Neurosurg. 2003, 17:60-64.

20. Borges A: "Trigeminal neuralgia and facial nerve paralysis". Eur Radiol. 2005, 15:511-33.

21. Jursinic PA, Rickert K, Gennarelli TA, Schultz CJ: "Effect of image uncertainty on the dosimetry of trigeminal neuralgia irradiation". Int J Radiat Oncol Biol Phys. 2005, 62:1559-67.

22. Stancanello J, Muacevic A, Sebastiano F, Modugno N, Cerveri P, Ferrigno G, Uggeri F, Romanelli P: "3T MRI evaluation of the accuracy of atlas-based subthalamic nucleus identification". Med Phys. 2008, 35:3069-77.

23. Maes F, Collignon A, Vandermeulen D, Marchal G, and Suetens P: "Multimodality image registration by maximization of mutual information". IEEE Trans. Med. Imaging . 1997, 16:187-98.

24. W Press: Numerical Recipes in C . Cambridge University Press, New York; 2003.

25. Ashburner J: Computational neuroanatomy. Ph.D. thesis. http:// www.fil.ion.ucl.ac.uktjohn/thesis.. 